\title{
$\mathrm{CiSj}$
}

\section{USE OF A COST-EFFECTIVE NEUROHEADSET EMOTIV EPOC FOR PATTERN RECOGNITION PURPOSES}

\author{
Aleksandra Kawala-Janik ${ }^{1)}$, Jerzy Baranowski ${ }^{2)}$, Michal Podpora ${ }^{1)}$, Pawel Piatek ${ }^{2)}$, \\ Mariusz Pelc ${ }^{1,3)}$ \\ ${ }^{1)}$ Faculty of Electrical Engineering, Automatic Opole University of Technology, \\ ul. Proszkowska 76, 45-756 Opole, Poland, \\ \{a.kawala-janik, m.podpora, m.pelc\}@po.opole.pl, http://www.we.po.opole.pl \\ ${ }^{2)}$ AGH University of Science and Technology, Faculty of Electrical Engineering, Automatics, \\ Computer Science and Biomedical Engineering, \\ al. Mickiewicza 30, 30-059 Krakow, Poland, \\ \{jb, ppi\}@agh.edu.pl, http://www.eaiib.agh.edu.pl \\ ${ }^{3)}$ School of Computing and Mathematical Sciences, University of Greenwich, Old Royal Naval College, \\ Park Row, SE10 9LS, London, United Kingdom, \\ m.pelc@greenwich.ac.uk, http://www.cms.gre.ac.uk
}

\begin{abstract}
Application of biomedical signals for the control purposes is currently growing interest of research society. Various biomedical signals enable various control prospects. In this paper application domain of using electroencephalographic signals obtained from an inexpensive Emotiv EPOC headset was described. It is also important to mention the possible implementation of the proposed method on an embedded platform, as it causes some significant limitations due to the little efficiency and low computing power of an embedded system platform. The proposed method enables to extend future application of the BCI system presented in this paper and it also gives more testing flexibility, as the platform can simulate various external environments. It is crucial to mention, that no filtering was done and that the traditional, statistical signal processing methods were in this work neither used, nor described. Copyright (C) Research Institute for Intelligent Computer Systems, 2014. All rights reserved.
\end{abstract}

Keywords: Electroencephalography (EEG), Brain-Computer Interface (BCI), signal processing, bio-informatics, control, robotics.

\section{INTRODUCTION}

Implementation of various biomedical signals in particular EEG - as an information source applied for the purpose of external environments control has become recently growing concern in the scientific world. Application of the EEG signals as a data source for Brain-Computer Interfaces (BCI) enables quick and direct communication between the computer (or any other device) and the brain [1].

Most of the BCI systems require expensive equipment with high computing power and it used complex signal processing methods, what eliminates the majority of the current solutions from their potential implementation on embedded platforms. This is because the analysis of biomedical signals and in particular EEG is very complex due the presence of various artifacts, which can be both internal and external. The signals itself are very sensitive to various disturbances and nonstochastic [2-4].
As these biomedical signals have non-stationary character, this may frequently lead to variation of SNR (Signal-Noise-Ratio), where low SNR can cause insufficient decoding accuracy and as a result affect the overall quality of a BCI system [3-5].

The novelty of the solution described in this work relies on application of basic mathematical operations, such as addition, subtraction, multiplication and division, only. Traditional, sophisticated signal processing methods were not applied. All experiments carried out for the study purpose were conducted in noisy, similar to real-life conditions [6].

\section{BRAIN-COMPUTER INTERACTION}

Last few decades have brought very thorough exploration of the BCI-related fields of research. BCI systems can be divided into two main groups invasive (with surgical implantation of electrodes) and non-invasive (e.g. those EEG-based, where no 
surgical intervention is made). It is also possible to divide the systems into the two varieties synchronous and asynchronous [1].

Any BCI system's goal is to record the brain activity in order to manage computer or machine actions [7]. In Fig. 1 the main six steps carried out in a typical BCI system were presented, where at the very beginning the cerebral activity is being recorded and then the data is extracted, classified and finally translated into commands, which enable to control a computer [7].

\section{BRAIN-COMPUTER INTERFACE}

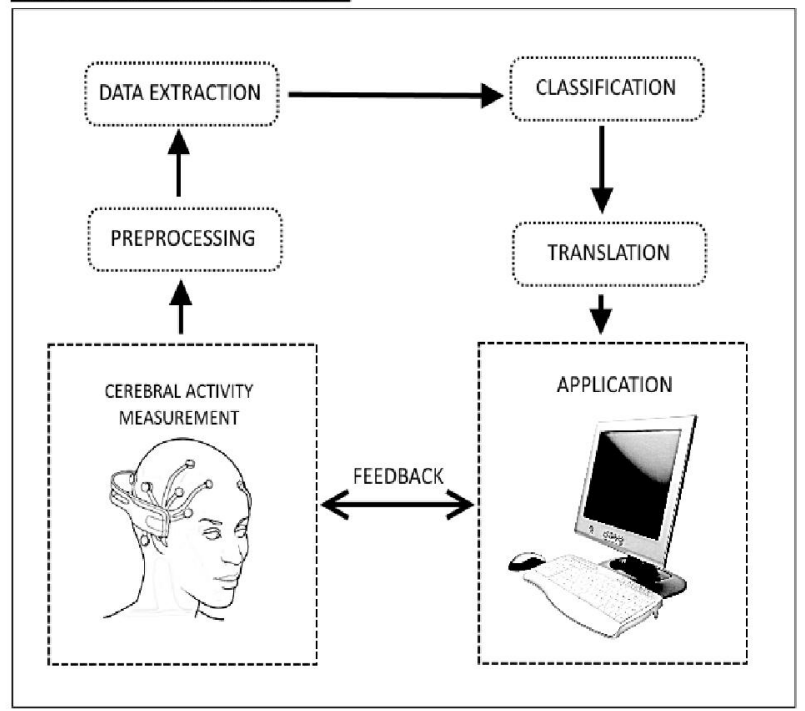

Fig. 1 - Six steps in a typical BCI system.

The BCI system presented in this work is based on EEG, where electrodes are placed on the surface of the scalp, which enables to read signals generated by the electrical activity of the brain $[7,8]$.

For this study purpose a non-invasive, EEG based Emotiv EPOC headset was applied. As it was important to use user-friendly, cost-effective, commercial headset (Fig. 2) $[6,8,9]$.

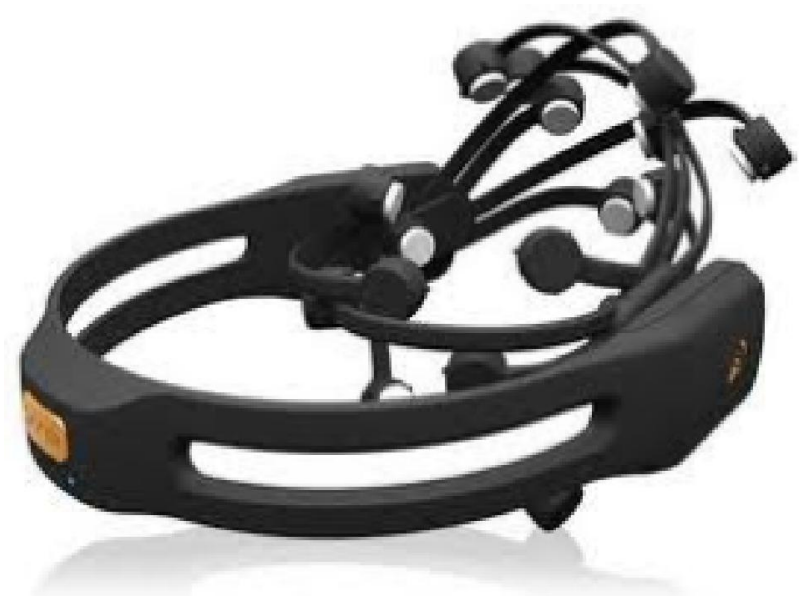

Fig. 2 - Neuroheadset Emotiv EPOC [9].

\section{RESEARCH METHODOLOGY}

As mentioned above - Emotiv EPOC headset was applied for this study purpose. This is a 14channel EEG-based headset [8-10]. It is also important to mention that most of the currently available solution require application of complex signal processing methods, which results in need of an expensive equipment due to the high computing power requirements. The method presented in this paper differs from other most common BCI systems by using basic mathematical operations only and does not require implementation of measurement equipment with high computing capability [6].

All experiments were conducted in similar to real life conditions, as only this would ensure that the proposed solution could be applicable in products such as wheelchair on crowded and noisy streets.

Twenty healthy, anonymous subjects participated in this study (Fig. 3), which had to imagine appropriate hand movement in accordance with the message appearing on the computer screen (Fig. 4). which played a role of a visual stimulus with all necessary instructions displayed on it.

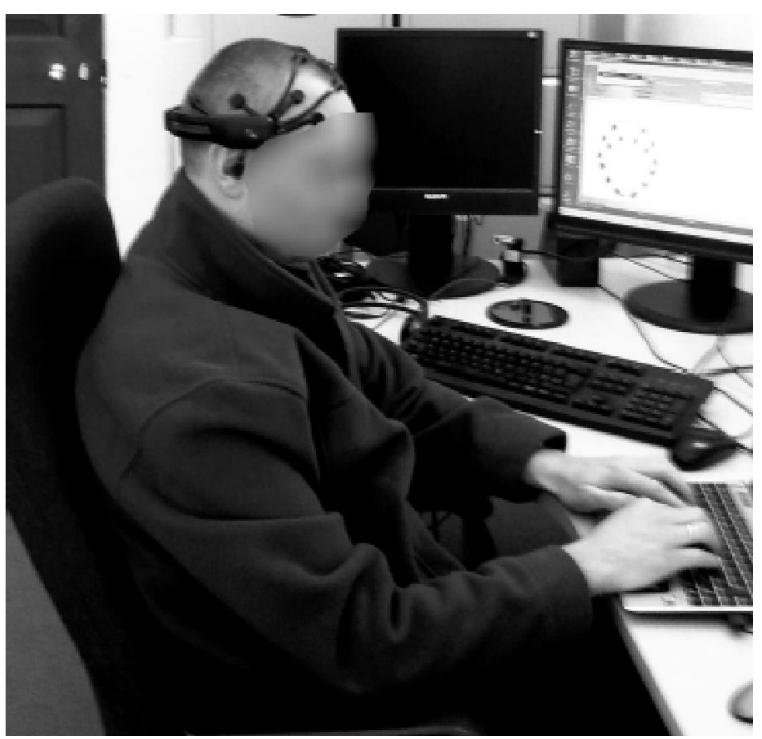

Fig. 3 - Anonymous research participant.

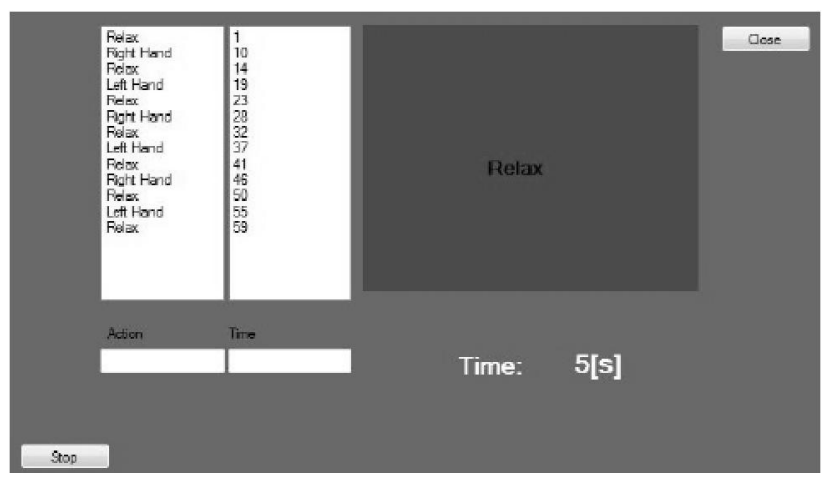

Fig. 4 - Visual stimulus with the tasks to be completed by research participants. 
The data was obtained from the two channels only - F3 and F4 (Fig. 5). The electrodes are placed according to the 10-20 electrodes placement standard. The data was recorded during imagery hand movements - right (F3 electrode) and left (F4 electrode).

Equipment used during this research is not a typical medical device and therefore does not register signals with medical precision.

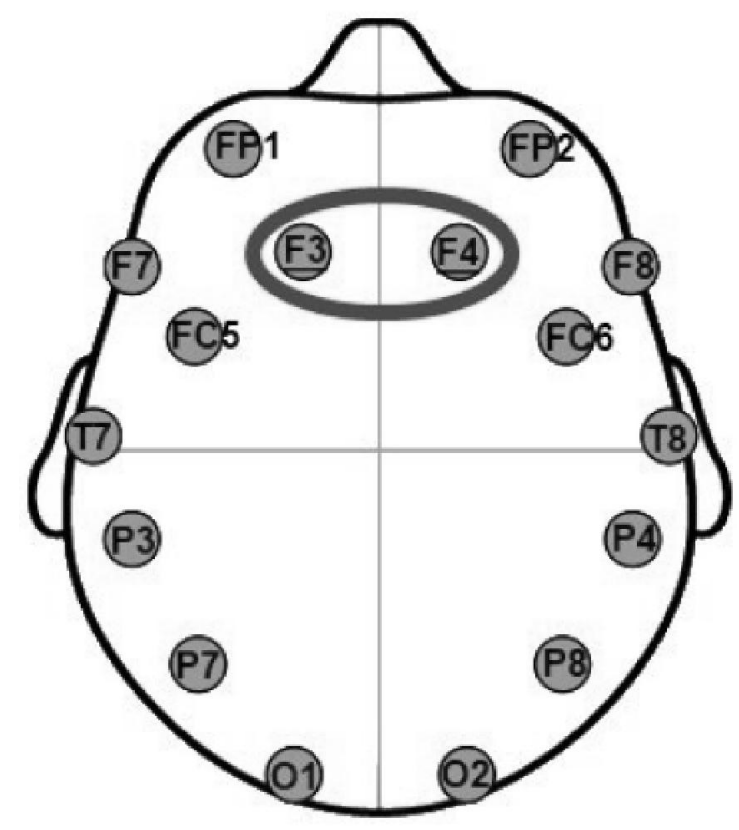

Fig. 5 - Electrodes placement.

The choice of an inexpensive, gaming equipment was made to present possible implementation of this sort of hardware in order to build cheap, but effective Brain-Computer Interface.

The Emotiv EPOC consists of 16 electrodes, where 14 only are placed on scalp and record EEG signal. The sampling rate of the device is $128 \mathrm{~Hz}$ and the bandwidth is between 0.2 and $45 \mathrm{~Hz}$, which is fair enough for the investigated frequency ranges $[9,10]$. It can also be successfully applied for recognition of user's emotions and has potentially wider use than traditional clinical electroencephalograph [11]. The neuro-headset uses three types of controls - EEG, EMG and Gyroscope $[12,13]$. It also has fewer scalp contacts than a typical professional device and potentially less accuracy. Also very little study was carried out in order to test its accuracy compared to the traditional EEG $[8,12,13]$.

\section{MATHEMATICAL INTERPRETATION OF THE PROPOSED METHOD}

As mentioned above - the method can only be presented with the use of basic mathematical operations only (1):
$\epsilon=\frac{(1-\alpha)}{N} \sum_{k=0}^{N-1}\left[\tilde{s}_{i}\left(k T_{s}\right)-\tilde{p}_{i}\left(k T_{s}\right)\right]^{2}+\frac{\alpha}{M} \sum_{l=0}^{M-1}\left[\left|\tilde{S}_{j}\left(l f_{s}\right)\right|-\left|\tilde{P}_{j}\left(l f_{s}\right)\right|\right]^{2}$,

where $t=k T_{s} \quad$ is the discrete time as $(k=0,1, \ldots, N-1), \quad \tilde{s}_{i}\left(k T_{s}\right)$ and $\tilde{p}_{i}\left(k T_{s}\right)$ for $i=1, \ldots, r$, are the discrete time representations of the ith signal and its pattern (or model depending on use), respectively, sampled at the frequency $F_{s}=\frac{1}{T_{s}}$, where $T_{s}$ is the sampling interval, $\left|\tilde{S}_{j}\left(l f_{s}\right)\right|$ and $\left|\tilde{P}_{j}\left(l f_{s}\right)\right|$ are the single-sided amplitude spectra of $\tilde{s}_{i}\left(k T_{s}\right)$ and $\tilde{p}_{i}\left(k T_{s}\right)$ respectively, with $f_{s}$ being the frequency steprelated to (but not necessarily equal) to $F_{s}$. The normalisation ensures that $\left(\tilde{s}_{i}, \tilde{p}_{i}, \tilde{S}_{j}, \tilde{P}_{j}\right) \in[0,1]$ and that the values of $C$ always belong to the $[0,1]$.

It is possible to differentiate two components of signal for the purpose of analysis. The weighted difference between the pattern and the signal is set up for both domains - the time domain and the frequency domain. In case the signal is of bad quality - very noisy, then - as a result - its timedomain representation may not be very useful for the research purposes. In this case the coefficient should be set to the value ' 1 ' or very close to ' 1 ', so that only the frequency domain component would be taken into account. Typically - as the most optimal solution - the value of the ' $\alpha$ ' coefficient should be set to '0.5', which means that the both components are equally important. The novelty of the diagnostic (or pattern recognition) approach adopted for the purpose of this research is an application of a threshold imposed on, which enable to make decisions on the quality of pattern recognition.

\section{EXPERIMENTAL RESULTS}

For this study purpose all signals were recorded in a noisy, full of disturbances, environment. Fig. 6 illustrated signals obtained from the 'F3'-electrode during right-hand imagery movement. The signals matched. Signals were recorded from two different male, adult subjects - Subject 3 and Subject 7. It is also important to mention, that only raw, unfiltered signals were processed. No filtering at all was done.

In Fig. 7 the same signals, but in a normalised, scaled - $[0,1]$ form were presented.

In both views it is possible to notice 'peaks' present in signals, what may be considered as potential artifacts. However the proposed method 
contains features of mean-square method. This means that this method has attributes of averaging the values and as a result - the eventual 'peaks', which may occur in signals will be eliminated.

The signals also visualy seem to differ strongly, however it is clearly noticable that they oscilate around similar values.
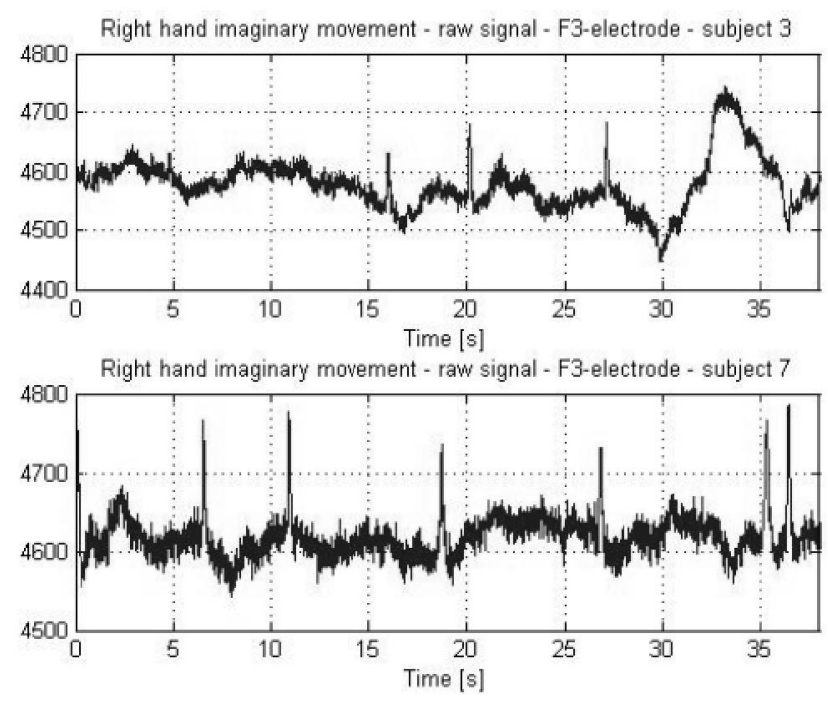

Fig. 6 - Subject 3 and 7 - imagery right-hand movement - 'F3'-electrode.

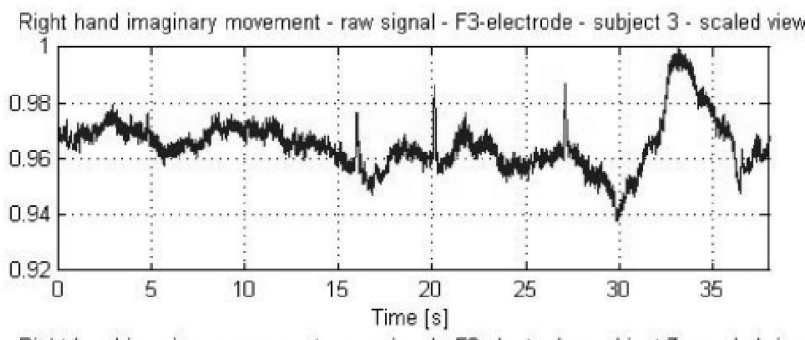

Right hand imaginary movement - raw signal - F3-electrode - subject 7 - scaled view

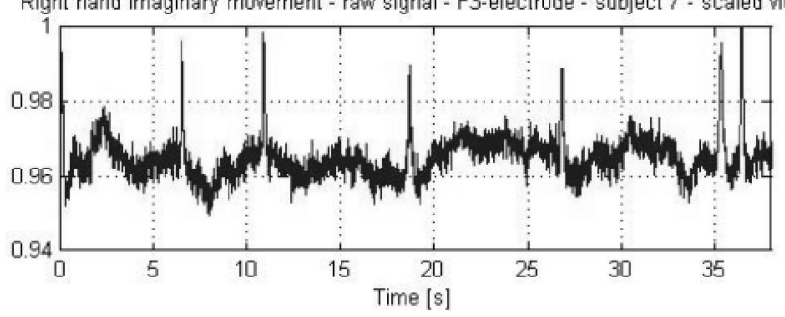

Fig. 7 - Subject 3 and 7 - imagery right-hand movement - 'F3'-electrode - normalised view.

\section{RESEARCH CHALLENGES}

All the above mentioned numeric procedures were carried out in MATLAB software package. The whole research was challenging due to the nonstationary nature of biomedical signals. Also implementation of the embedded platform has caused significant limitations in choosing appropriate signal processing method. As it also precludes application of advanced neural networks, as the traditional embedded platform's micro controller would not be able to proceed the computing. The conducted research was carried out on a small (for statistic criteria) group of subjects and therefore the obtained results could be unreliable, however it could be consider as a preliminary study (similar to: $[14,15]$ ).

Analysis of efficiency of the proposed solution for the particular candidates has not been done at this stage. As the sophisticated signal processing methods cannot be used as it may cause prohibitive computational burdens. Also the device itself (Emotiv EPOC headset) had some disadvantages, as it was not designed for clinical usage. The obtained signals did not contain full information unlike it is in case the signals are recorded with a typical electroencephalograph. As a result - the recorded EEG signals had also a very low accuracy.
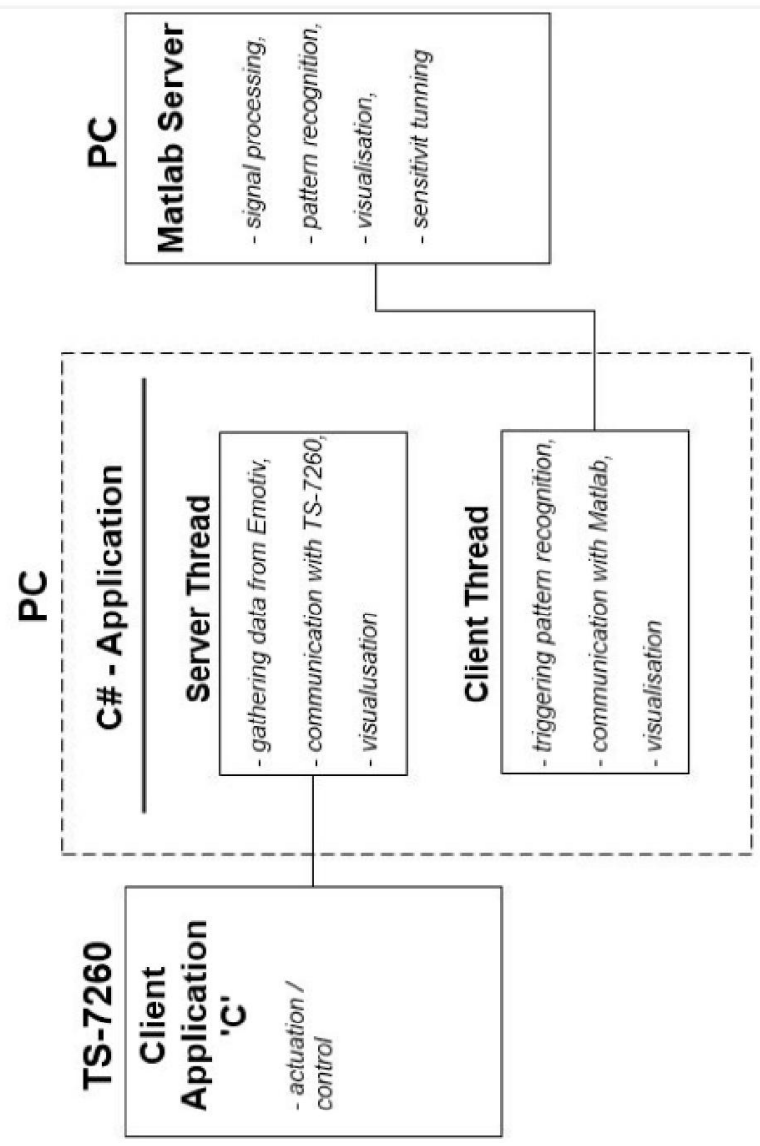

Fig. 8 - Scheme of the communication between the particular BCI components.

As the current approach is based on the fast prototyping scheme. Testbed was based on communication between PC and TS-7260 (embedded platform) and between MATLAB and PC. The main aim of this work was to build a fully working, standalone BCI system with no need of using Matlab or PC. The structure of the BCI system fast prototyping testbed described in this paper was presented in Fig. 8. 
In future applications it will be desired to construct a simple embedded system based on a mobile computing platform. Main approach, that is considered is the automatic code generation directly from MATLAB/Simulink with use of Simulink Coder and Matlab Coder toolboxes (http://www.mathworks.com/products/matlabcoder/) [27].

\section{CONTRIBUTION OF THE RESEARCH}

In this study traditional complex statistical signal processing methods were not involved. The novelty of the proposed solution relies on application of the basic mathematical operations.

The proposed method is simple, novel and what is the most important - efficient. No filtering was done as it did not improve the results (some of the pilot study was done in order to test the effectiveness of filtering - see: [26].

It is also important to mention, that the Emotiv EPOC provides wireless USB connector and has relatively good battery life - up to 12 hours work [16]. The signals recorded with Emotiv EPOC headset are quite noisy [17]. Also - as already mentioned above - Emotiv EEG (or EPOC) is an inexpensive, non-invasive, 'off-the-shelf' wireless EEG neuroheadset, where the raw data stream is locked. Also the recording accuracy is low, however it enables successful implementation in various BCI applications [28]. All the numeric procedures of this work were conducted in MATLAB.

Adopted tools for signal processing could be more sophisticated, although it might led to prohibitive computational burdens, in particular in the embedded system environment selected owing to the lowcost implementation prerequisite.

Also the implementation of Emotiv EPOC headset had some disadvantages, as the device was not used for clinical applications and therefore the accuracy of the registered signal was not very high, however the conducted initial tests proved efficiency and suitability of the implementation of the proposed solution in real-life environments.

As it was mentioned above, equipment used for the research purposes was not designed for clinical use. It is inexpensive and easy to use (also for inexperienced potential user) $[1,18,19]$. The proposed device became very popular recently among other BCI researchers due to its intuitive user interface and low price $[18,19]$.

Other BCI solutions (eg. Khushaba - [21], Volosyak - [22] or Cholula - [23]) are based on analysis of various brain-signals, such as $\alpha$ or $\beta$ unlike the proposed by the authors of the hereof paper method, where only the waves are being processed.
Many scientists engaged in analysis of EEG signals state that the frequencies of: $\delta, \theta$ and $\alpha$ are strongly correlated with drowsiness, fatigue and poor tasks performance [29].

The above mentioned methods also require high computing power, what makes it impossible to implement in traditional embedded platforms.

The novelty of the described method relies also on its simplicity and lack of traditional statistic methods, applied in other BCI systems (e.g. [21-23). For the research purposes only two electrodes were taken into consideration, and as a result only two channels have been used - 'F3' and 'F4' [22].

The proposed method's efficiency is quite high $91.7 \%$ in case signal was gathered in quiet environment, recorded during left hand movement from the electrode placed on 'F4' position, which was suprisingly high. However in case the same signals, from the same electrode were gathered in different conditions - noisy environment - the efficiency dropped to $86.7 \%$. For the signals (both noisy and quiet environment) obtained during righthand movement and recorded from the 'F3' electrode - the efficiency was the same $-86.7 \%$. Traditional methods - SSVEP (Steady-State Visual Evoked Potential) or P300 Paradigm oscillate between $69.2 \%$ and $100 \%$ and require higher computing power $[15,22,23]$. SSVEP BCI solution's overall pattern-recognition efficiency (presented in: [15]) was $84 \%$, which is much lower than the effectivness of the solution proposed by the authors of this paper. Also both SSVEP and P300-based BCIs require implementation of complex signal processing method and therefore would not be suitable for the method used by authors of this publication [15, 23].

Also no filtering was done in this work, as some initial tests have proven that in analysis of signals with limited information data (when the signals have been hardware-preprocessed) filtering 'cuts-off' also the valuable information, what significantly decreases the overall signal processing performance $[6,10,24]$.

Also various filtering methods such as adaptive spatio-temporal (AST) algorithms are frequently applied. The advantage of such algorithm is that it reduces the danger of signals overfitting by constructing a low-pass temporal filter with the implementation of two-parameter Gaussian kernel [30]. Using such a sophisticated method, which would also involve some mathematical modelling is was impossible to implement on the embedded platform with a very limited computing.

To sum it all up - the novelty of the proposed solution (despite its simplicity and limitations) relies on repudiation of the traditional signal processing methods based on complex statistics. The use of basic mathematical operations enables potential 
implementation of the methods in embedded systems with small computing power. The method can be also easily transferred into any programming language - including ' $\mathrm{C}$ ' or 'Assembler'. During very thorough literature studies similar method have not been found.

\section{FUTURE WORK}

Further research will be carried out in three main areas. The first one, considers the algorithmic and conceptual improvements. The signal processing algorithm has a potential for development, with advanced techniques. Especially methods of filtering will be reconsider with possible applications of statistical filtering via densitogram [25] or Bayesian filtering [26].

Also pattern recognition and classification algorithms require additional analysis. Currently field is dominated by neural based approaches, which have many drawbacks. Authors consider application of Bayesian classifiers. In order to verify any potential advance in algorithm modification series of additional experiments is needed.

Second area for improvement is focused on hardware realisation of the system. In particular two basic approaches are considered. The first one is to construct a dedicated microcontroler system which will be responsible for data processing and interpretation. This approach has its merits, especially if true real time requirements are present. This is where automatic code generation shines the most, as Simulink algorithms can be smoothly implemented. Main drawbacks are limits to computing power and the requirement of creation of dedicated system, which in low numbers is simply not cost effective. Different approach is to use a mobile embedded platform such as smartphone. In such situation actual electronic circuits are limited to some kind of wireless interface - for example for Bluetooth or WLAN. In such case, smartphone acquires data from the interface and takes care of the processing. What is especially interesting, because of cellular network there is a constant internet access and the potential for cloud computing. It is currently used in such applications like Google Voice Search.

Third avenue for development is the extension of the system by additional methods of biomedical signal acquisition. In particular speech, EMG and EOG. Potential for integration of such systems is unlimited with broad area of applications.

The further study will focus also on improvement of the signal-processing method and application of other bio-signals - in order to extend the possible applicability and ameliorate its effectiveness. It will also involve improvement of the proposed algorithm in order to improve the pattern recognition efficiency. There are also plans for conduction more experiments with the implementation of other inexpensive, easily available on market headsets in order to obtain more data and to make the proposed method more reliable.

As mentioned already above, all numeric procedures were carried out in MATLAB. The further research plans involve building a standalone system, where not only EEG signals will be used, but also other bio- signals such as speech, EMG and EOG.

Research will also be conducted on wider group of subjects in order to make the obtained results more reliable.

Some initial tests (Fig. 9) were already run on adapting the proposed method for EMG signals recorded during simple finger movements. The efficiency of the method was in that case a little bit lower - ca. 85\%. Also some initial research regarding simultaneous on-line analysis of EEG and fMRI signals was already conducted [31-33].

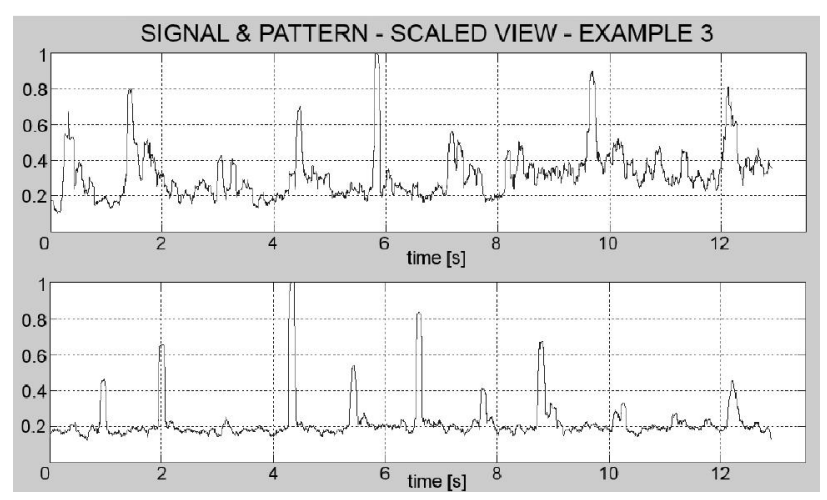

Fig. 9 - Sample of the left (top) and right (bottom) index fingers movements.

\section{REFERENCES}

[1] Wenjia Ouyang, Kelly Cashion, Vihayan K. Asari, Electroencephalograph based brain machine interface for controlling a robotic arm, 2013 IEEE (AIPR) Applied Imagery Pattern Recognition Workshop: Sensing for Control and Augumentation, (October 23-25, 2013), pp. 1-7.

[2] K. Blinowska, J. Zygierewicz, Practical Biomedical Signal Analysis Using MATLAB, Series in Medical Physisc and Biomedical Engineering, CRC Press, Taylor and Francis Book, 2012.

[3] S. Gao, Y. Wang, X. Gao, B. Hing, Visual and auditory Brain-Computer Interfaces, IEEE Transactions on Biomedical Engineering, (Accepted for Publication) (2014).

[4] H. I. Suk, S. W. Lee, A novel Bayesian framework for discriminative feature extraction 
in Brain-Computer Interfaces, IEEE Transactions on Pattern Analysis and Machine Intelligence, (35) 2 (2013), pp. 286-299.

[5] D. Huang, K. Qian, D.-Y. Fei, W. Jia, X. Chen, Electroencephalography (EEG)-based BrainComputer Interface (BCI): A 2-D virtual wheelchair control based on Event-Related Desynchronization/Synchronization and state control, IEEE Transactions on Neural Systems and Rehabilitation Engineering, (20) 3 (2012), pp. 379-388.

[6] Aleksandra Kawala-Janik, Michal Podpora, Mariusz Pelc, Jerzy Baranowski, Pawel Piatek, Implementation of an inexpensive EEG headset for the pattern recognition purpose, 2013 IEEE (IDAACS) 7th International Conference on Intelligent Data Acquisition and Advanced Computing Systems, (Berlin, September 12-14, 2013), pp. 399-403.

[7] Simon Grude, Matthew Freeland, Chenguang Yang, Hongbin Ma, Controlling mobile Spykee robot using Emotiv Neuro Headset, 2013 (CCC) 32nd Chinese Control Conference, (Xi'an, July 26-28, 2013), pp. 5927-5932.

[8] Hidenori Boutani, Mieko Ohsuga, Applicability of the "Emotiv EPOC Neuroheadset" as a userfriendly input interface, 2013 (EMBC) 35th Annual International Conference of the IEEE Engineering in Medicine and Biology Society, (Osaka, July 3-7, 2013), pp. 1346-1349.

[9] Official website of the Emotiv Inc. Company: http://www.emotiv.com

[10] Emotiv Wikia Page: http://www.emotiv.wikia. com

[11] R. Bernays, J. Mone, P. Yau, M. Murcia, J. Gonzales-Sanchez, M. E. ChavezEcheagaray, R. Christopherson, R. Atkinson, Lost in the dark: Emotion adaptation, 2012 (UIST) 25th Annual ACM Symposium on User Interface Software and Technology.

[12] G. M. Poor, L. M. Leventhal, S. K. Bowling, J. Ringenberg, S. D. Jaffee, Thought Cubes: Exploring the use of an inexpensive BrainComputer Interface on a mental rotation task, 2011 (ASSETS) 13th International ACM SIGACCESS Conference on Computers and Accessibility.

[13] C. Vi, S. Subramanian, Detecting Error-related negativity for interaction design, 2012 (CHI) SIGCHI Conference on Human Factors in Computing Systems, ACM.

[14] A. Vourvopoulos, F. Liarokapis, Braincontrolled NXT robot: Tele-operating a robot through brain electrical activity, $20113 \mathrm{rd}$ International Conference on Games and Virtual Words for Serious Applications.
[15] O. Friman, I. Volosyak, A. Graeser, Multiple channel detection of Steady-State Visual Evoked Potentials for Brain-Computer Interfaces, IEEE Transactions on Biomedical Engineering, (54) 4 (2007).

[16] J.-M. Seigneur, The Emotional Economy for the Augmented Human, $(A H)$ 2nd Augmented Human International Conference, ACM, 2011.

[17] A. T. Campbell, T. Choudhury, S. Hu, H. Lu, M. K. Mukerjee, M. Rabbi, R. D. S. Raizada, NeuroPhone: Brain-Mobile Phone Interface. Using a Wireless EEG Headset. 2010 (MobiHeld) 2nd ACM SIGCOMM workshop on Networking, Systems, and Applications on Mobile Handhelds, USA (August 2010).

[18] M. van Vliet, A. Robben, N. Chumerin, N. V. Manyakov, A. Combaz, M. M. van Hulle, Designing a Brain-Computer Interface Controlled Video-Game Using Consumer Grade EEG Hardware. 2012 (BRC) Biosignals and Biorobotics Conference, Brazil (January 2012).

[19] A. Luo, J. Sullivan, A User-Friendly SSVEPBased Brain-Computer Interface Using a TimeDomain Classifier, Journal of Neural Engineering, (7) 2 (2010).

[20] Y. Liu, X. Jiang, T. Cao, F. Wan, P. Un Mak, P.-I. Mak, M. I. Vai, Implementation of SSVEP Based BCI with Emotiv EPOC. 2012 (VECIMS) IEEE International Conference on Virtial Environments Human-Computer Interfaces and Measurement Systems, China (July 2012).

[21] R. N. Khushaba, S. Kodagoda, G. Dissanayake, L. Greenacre, S. Burke, J. Louviere, A Neuroscientific Approach to Choice Modeling: Electroencephalogram (EEG) and User Preferences, 2012 (IJCNN) the 2012 International Joint Conference on Neural Networks.

[22] I. Volosyak, Ch. Guger, A. Graeser, Toward BCI Wizard - Best BCI Approach for Each User, 2010 (EMBS) the 32nd Annual International Conference of the IEEE EMBS, Argentina, (August-September 2010).

[23] G. Rosas-Cholula, J. M. Ramirez-Cortes, V. Alarcon-Aquino, J. Martinez-Carballid, P. Gomez-Gil, On Signal P-300 Detection for BCI Applications Based on Wavelet Analysis and ICA Preprocessing, 2010 Electronics, Robotics and Automotive Mechanics Conference.

[24] A. Kawala-Janik, Efficiency Evaluation of External Environments Control Using BioSignals, (PhD Dissertation), The University of Greenwich, 2013. 
[25] J. Baranowski, P. Piatek, A. Kawala-Janik, M. Pelc, R. J. Anthony, Application of Kernel Density for Analysis of EEG Signals, Ubiquitous Computing and Ambient Intelligence, Lecture Notes in Computer Science, (7656) 2012, pp 403-406.

[26] L.G. Bretthorst, Bayesian Spectrum Analysis and Parameter Estimation, Lecture Notes in Statistics, (48) 1988, Springer Verlag, New York.

[27] P. Piatek, D. Marchewka, M. Pauluk, Automatyczna generacja kodu regulatora dla wbudowanego sterownika ukladu magnetycznej lewiacji, Projektowanie, analiza i implementacja systemow czasu rzeczywistego (red. L. Trybus i S. Samolej), WKL, Warszawa, 2011, pp. 187-196. (in Polish)

[28] N. Al-Ghamdi, G. Al-Hudhud, M. Alzamel, A. Al-Wabil, Trials and tribulations of BCI control applications, Science and Information Conference (SAI) 2013, pp. 212-217.

[29] Ch.-T. Lin, Ch.-H. Chuang, Ch.-Sh. Huang, Sh.-F. Tsai, Sh.-W. Lu, Y.-H. Chen, L.-W. Ko, Wireless and Wearable EEG Systems for Evaluating Driver Vigilance, IEEE Transactions on Biomedical Circuits and Systems, vol. 8, no. 2, 2014, pp. 165-176.

[30] J. Lu, K. Xie, D. McFarland, Adaptive SpatioTemporal Filtering for Movement Related Potentials in EEG based Brain-Computer Interfaces, IEEE Transactions of Neural Systems and Rehabilitation Engineering, Accepted for Publictation in 2014. DOI: 10.1109/TNSRE.2014.2315717.

[31] X. Hongjing, R. Dan, M. S. Cohen, Coupled basis learning and regularized reconstruction for BCG artifact removal in simultaneous EEGfMRI studies, 2013 IEEE 10th International Symposium on Biomedical Imaging (ISBI), 2013, pp. 986-989.

[32] A. Kawala-Janik, M. Podpora, M. Blaszczyszyn, J. Kolanska-Pluska, B. Grochowicz, Combined fMRI- and EEGbased Brain-Computer Interface - Pilot Study, XXXVII International Conference on Fundamentals of Electrotechnics and Circuit Theory 2014, p. 72.

[33] A. Kawala-Janik, M. Podpora, J. KolanskaPluska, M. Konieczny, B. Grochowicz, Pilot Study on On-Line Analysis of Biomedical Signals Using Labview, XXXVII International Conference on Fundamentals of Electrotechnics and Circuit Theory 2014, p. 73.

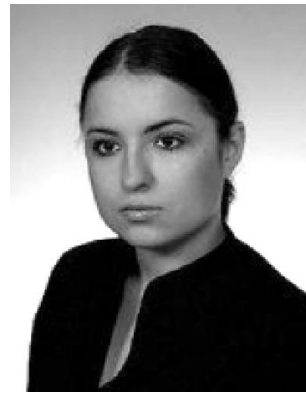

Aleksandra Kawala-Janik, Ph.D. received M.Sc. in Computer Engineering from the Opole University of Technology in Poland in 2007. In 2012 she has completed doctorate in Computing and was awarded in 2013 Ph.D. degree in Computing by the University of Greenwich in London. She has been working as a Lecturer in both Opole University of Technology and University of Greenwich. She has also gained some industrial experience. Currently she is employed as assistant professor at the Department of Robotics and Applied Informatics, Institute of Electromechanical Systems and Industrial Electronics, Faculty of Electrical Engineering, Automatic Control and Informatics, Opole University of Technology. Her research interests include BrainComputer Interfaces, Human-Computer Interaction, Biomedical Signal Processing and Articial Intelligence.

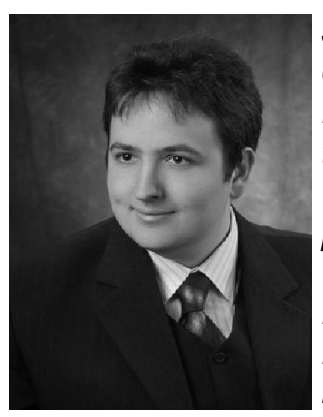

Jerzy Baranowski, Ph.D. He obtained MSc in Control Engineering in 2006 and $P h D$ in technical sciences in 2010. Currently he is an assistant professor in AGH University of Science and Technology at the Department of Automatics and Biomedical Engineering. His main research interests are control theory, Bayesian statistics, numerical methods and optimization.

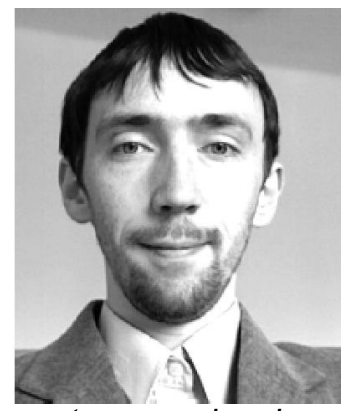

Michal Podpora, Ph.D. received B.Sc. (2002) and M.Sc. (2004) degrees in Computer Engineering from the Opole University of Technology in Poland, where he was also awarded in 2012 (Ph.D. in Control Engineering and Robotics). Until 2010 he was employed in industry sector as a developer of research software and as a EU-funds fundraiser. Since 2010 he was Research Department Manager and Project Manager of cofinanced research project in industry. At the same time (2005-2011) he was employed as K-12 IT teacher. He is also Apple Distinguished Educator. He has been employed as associate professor at the Faculty of Electrical Engineering, Automatic Control and Informatics, Opole University of Technology since 2012. His main research interests include machine vision, vision reasoning, cognitive systems, robotic systems and distributed systems. 


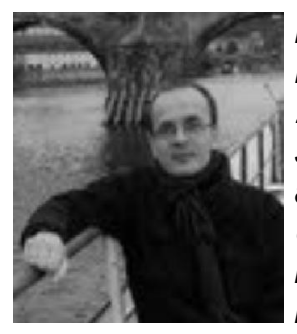

Pawel Piatek, Ph.D. He obtained MSc in Control Engineering in 2000 and PhD in technical sciences in 2007. Currently he is an assistant professor in $A G H$ University of Science and Technology at the Department of Automatics and Biomedical Engineering. His main research interests are embedded systems, signal processing and discrete control.

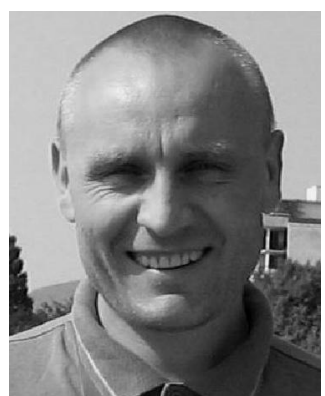

Mariusz Pelc, Ph.D. is currently employed at the University of Greenwich, School of Computing and Mathematical Sciences, Smart Systems Technology Department and also at the Opole University of Technology, Faculty of Electrical Engineering, Automatic Control and Computer Science. His scientific activities are spread across various domains, including automatic control and robotics, modern computer / embedded (control) systems, software systems as well as computer clusters and parallel processing. His most recent scientific activity is focused on autonomic and self-* systems and most of all - validation and verification of such systems, interoperability issues and certification of self-* systems. He has published in total of nearly 50 research papers and 2 books. 